Supporting Information for

\title{
One-step controlled synthesis of size tunable toroidal gold particles for biochemical sensing
}

Phuong-Diem Nguyen ${ }^{l}$, Xuanru Zhang ${ }^{l}$ and Judith $\mathrm{Su}^{1,2 *}$

${ }^{1}$ Department of Biomedical Engineering, The University of Arizona, Tucson, AZ 85721

${ }^{2}$ Wyant College of Optical Sciences, The University of Arizona, Tucson, AZ 85721

\section{Corresponding Author}

Corresponding Author’s Email: judy@optics.arizona.edu, Phone: (+1) 520-621-4240. 
(a)

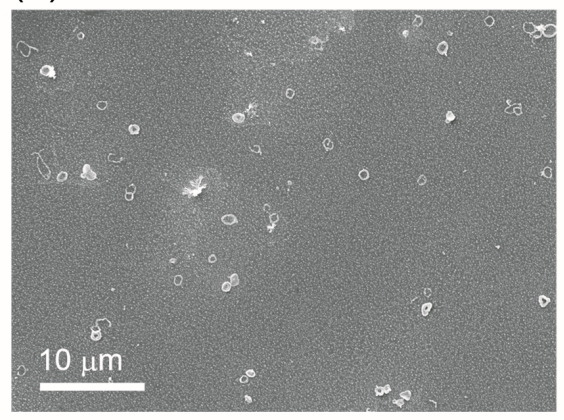

(b)

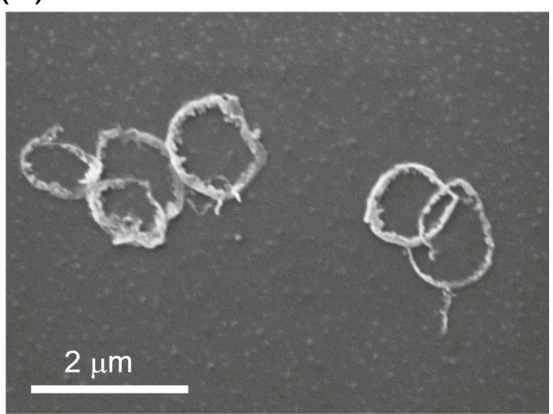

Figure S1. SEM images of tAUP synthesized at $40{ }^{\circ} \mathrm{C}$ ( $\mu \mathrm{m}$-tAUPs). (a) $\mu \mathrm{m}$-tAUPs. (b) A close look at the $\mu \mathrm{m}$-tAUPs showing nanospike structures protruding inwards from the toroidal loop. 
(a)

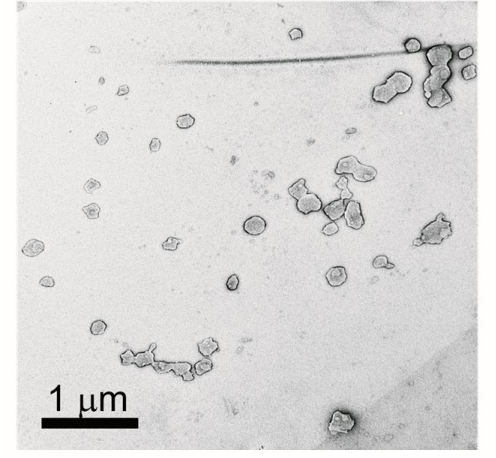

(b)

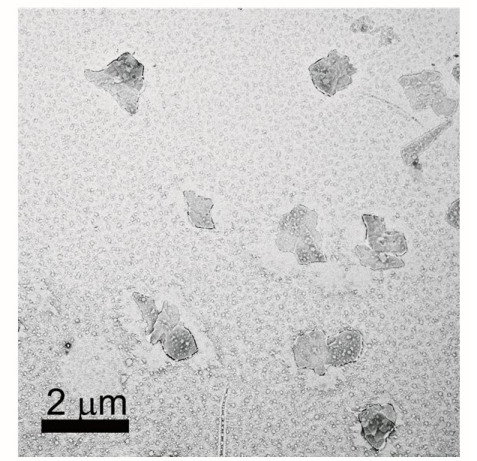

Figure S2. TEM image of SDS/CTAB templates formed at different temperatures. The morphology of formed templates was visualized by uranyl acetate staining (a) At $21^{\circ} \mathrm{C}$, (b) At 40 ${ }^{\circ} \mathrm{C}$. 
(a)

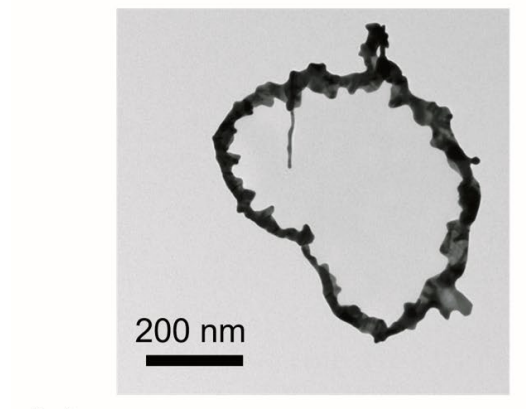

(c)

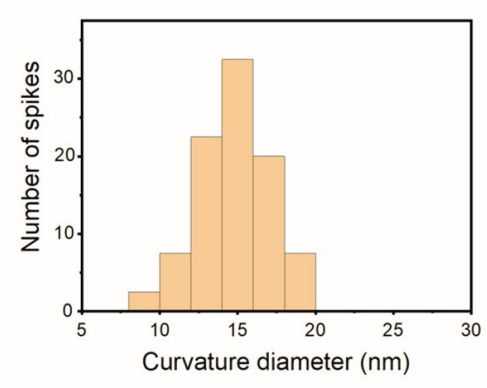

(b)
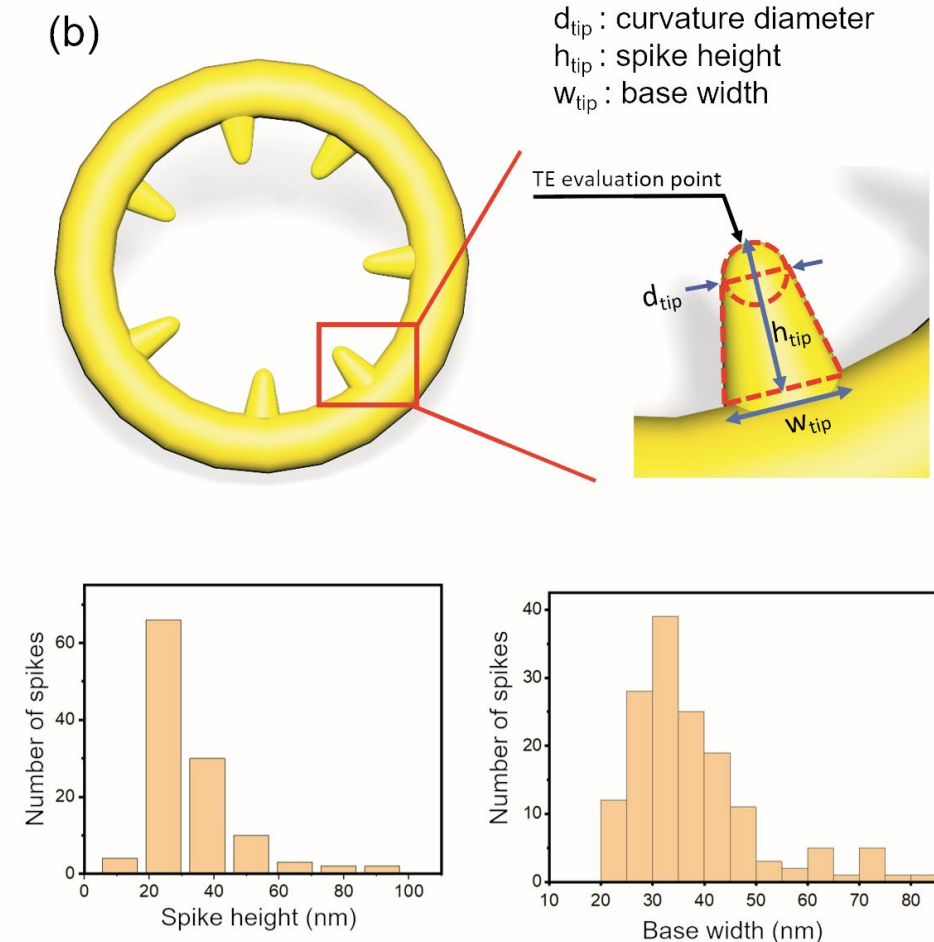

$\mathrm{d}_{\text {tip }}$ : curvature diameter

$h_{\text {tip }}:$ spike height

$\mathrm{W}_{\mathrm{tip}}$ : base width

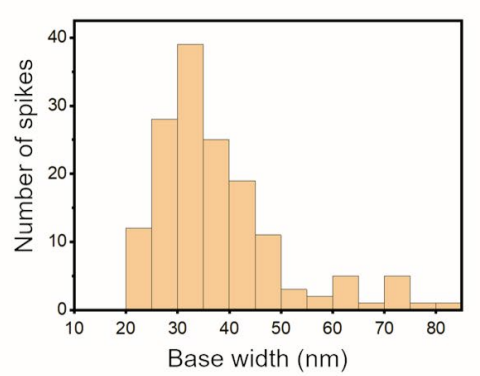

Figure S3. Dimension analysis of nanospike generated on tAUP structures characterized by TEM imaging and analyzed by ImageJ software (a) A representative TEM image of a single tAUP generating multiple nanospikes, (b) 3D modeling of a single tAUP with nanospikes protruding inwards, the ring was modeled as an enclosed toroidal shape, the spike was modeled as eccentric cones with hemispherical tips, ${ }^{1,2}$ where $d_{\text {tip }}$ is the curvature diameter, $h_{\text {tip }}$ is the average height of nanospikes, $\mathrm{w}_{\text {tip }}$ is the average base width of the nanospikes, (c) Size distribution of the average $\mathrm{d}_{\text {tip }}, \mathrm{h}_{\text {tip }}, \mathrm{w}_{\text {tip }}$ values experimentally analyzed using ImageJ software. Results are from approximately 100 spikes which appeared normal to the electron beam. 


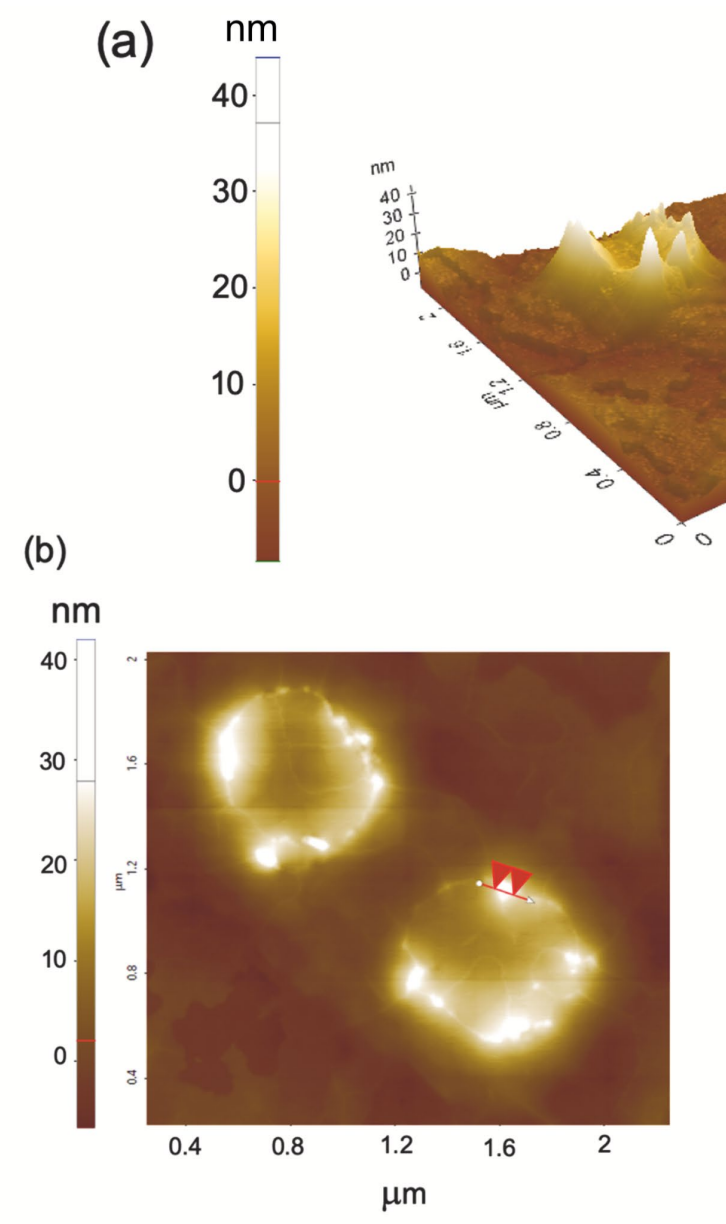

(c)

Height $(\mathrm{nm}) \quad$ Line Profile

(d)

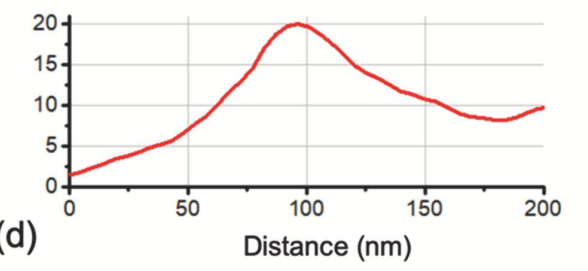

Pixel

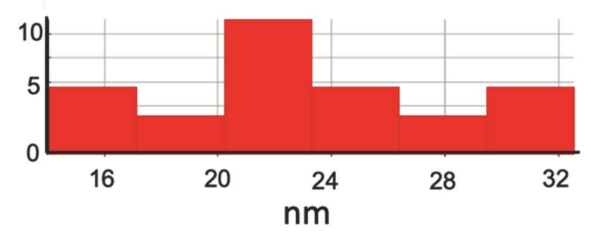

Figure S4. tAUP nanospike characterization using Atomic Force Microscopy (AFM) imaging of nmtAUP structure (a) 3D-topography of two nm-tAUPs taken in a $2.5 \times 2.5 \mu \mathrm{m}$ region, showing the roughness of the ring structure, (b) Topography of the tAUPs shown in (a). The line profile corresponds to the height profile of a nanospike protruding inward from the ring. (c) corresponding height profile from (b). (d) histogram of height (z-direction) taken from several spikes. 
(a)

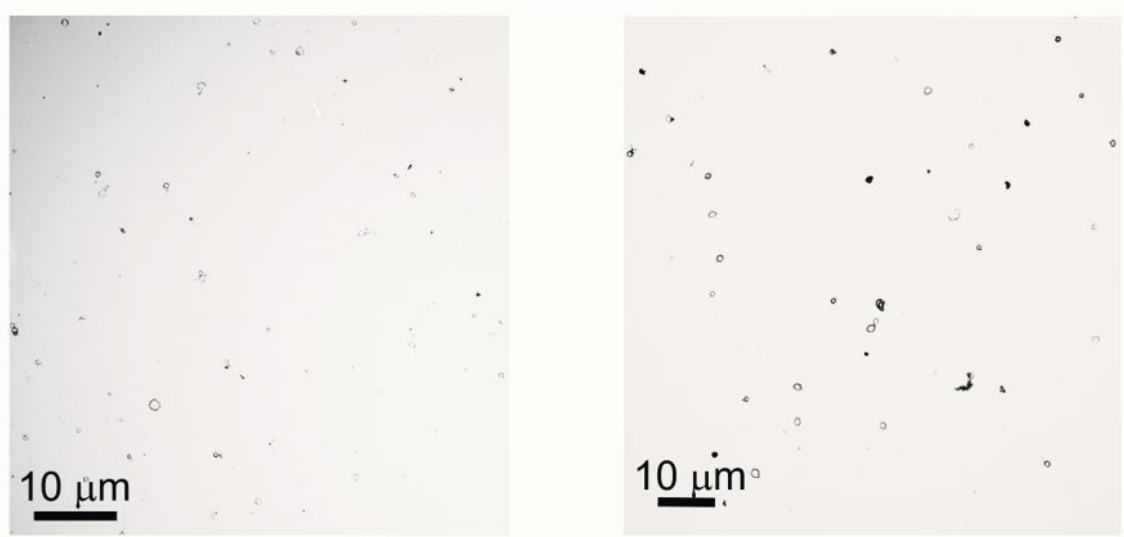

(c) (b)

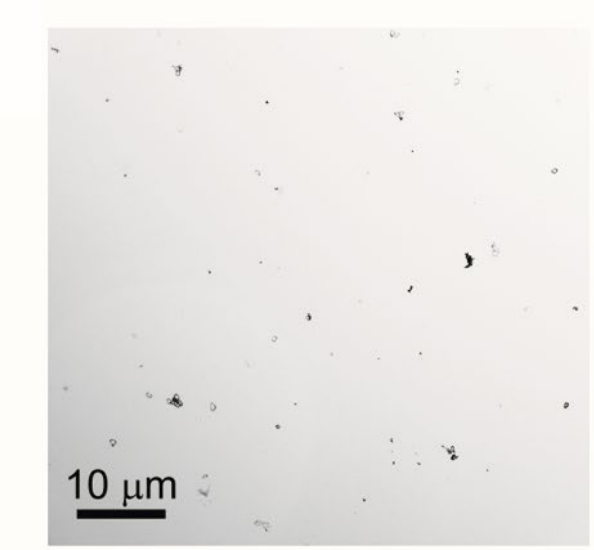

Figure S5. TEM images of toroidal gold particle (tAUP) synthesized at elevated temperatures (a) At $21^{\circ} \mathrm{C}$, (b) At $40{ }^{\circ} \mathrm{C}$, (c) At two step-temperature change from $4{ }^{\circ} \mathrm{C}$ to $21^{\circ} \mathrm{C}$. 


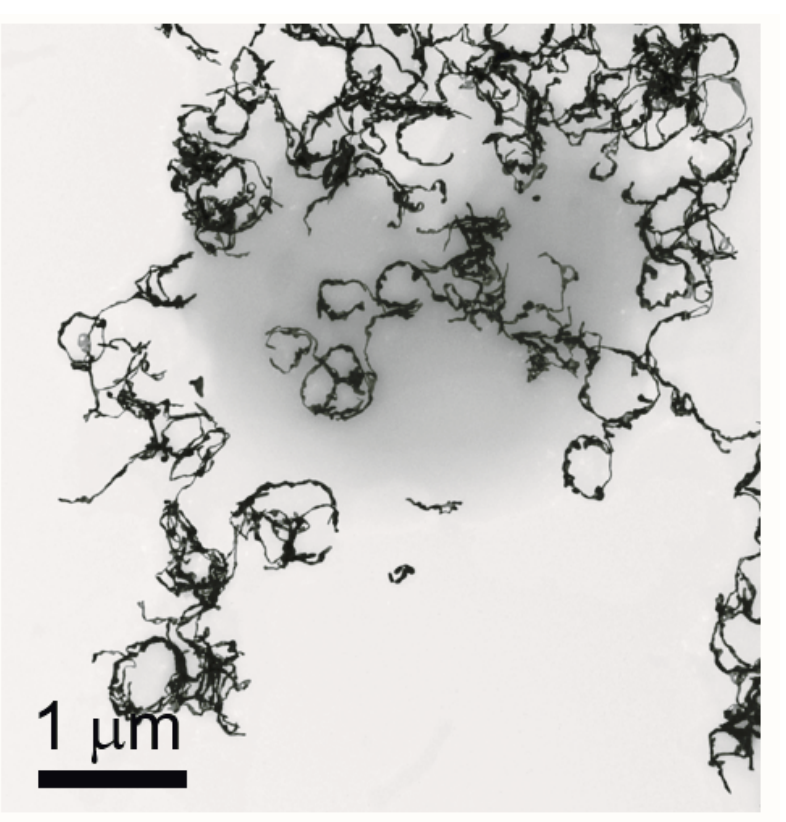

Figure S6. TEM image of tAUP synthesized without magnetic stirring. 


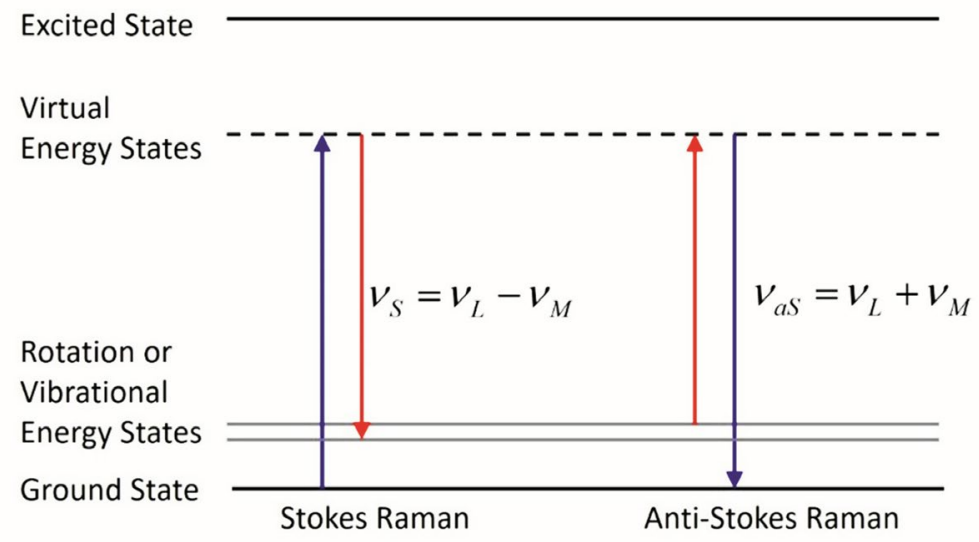

Figure S7. Schematic depiction of Stokes and anti-Stokes Raman scattering.

For Raman scattering,

$$
\begin{gathered}
v_{S}=v_{L}-v_{M} \\
v_{a S}=v_{L}+v_{M}
\end{gathered}
$$

in which $v_{\mathrm{L}}$ is the laser frequency, $v_{\mathrm{M}}$ is the molecule vibration or rotation frequency, and $v_{\mathrm{S}}$ (aS) is Stokes (Anti-Stokes) Raman frequency. The intensity of Raman signal $I_{R}\left(v_{\mathrm{R}}\right)$ equals:

$$
I_{R}\left(v_{R}\right)=N \sigma I_{\text {Excitation }}\left(v_{L}\right),
$$

in which $N$ is the number of scatters at the excitation laser spot, $\sigma$ is the scattering cross section and $I\left(v_{\mathrm{L}}\right)$ is the intensity of the excitation laser. Consistent to the definition in the experiment in the main text, the intensities of $I_{R}$ and $I_{\text {Excitation }}$ should be in unit of photons per area per second, proportional to power per area. If there are some near-field enhancement effects, the enhancement affects both the excitation and the Raman signal. The field enhancement $L$ at frequency $v$ is:

$$
L(v)=\left|\mathbf{E}_{\text {loc }}(v)\right| /\left|\mathbf{E}_{0}\right|,
$$

where $\mathbf{E}_{0}$ is the electric field amplitude of the excitation laser, and $\mathbf{E}_{\text {loc }}$ is the local electric field with the near-field enhancement effects. The intensity enhancement is the square of the field enhancement and applies to both the excitation intensity and emission cross-section:

Thus the enhanced Raman power will be:

$$
\begin{aligned}
I\left(v_{L}\right) & \rightarrow L^{2}\left(v_{L}\right) I\left(v_{L}\right), \\
\sigma_{R S} & \rightarrow L^{2}\left(v_{S}\right) \sigma_{R S} .
\end{aligned}
$$

$$
P_{S}\left(v_{S}\right)=N \sigma_{R S} L\left(v_{L}\right)^{2} L\left(v_{S}\right)^{2} I\left(v_{L}\right) .
$$

The enhancement will be:

$$
L\left(v_{L}\right)^{2} L\left(v_{S}\right)^{2} .
$$

As the molecule vibration and rotation frequencies are always much lower than the laser and Raman signal frequencies, the near-field enhancement at the laser and Raman signals are approximately equal: $\left|L\left(v_{L}\right)\right| \approx\left|L\left(v_{S}\right)\right|$. 
Thus, the enhancement will be:

$$
L\left(v_{\mathrm{L}}\right)^{4}=\frac{\left|\mathbf{E}_{\mathbf{l o c}}\right|^{4}}{\left|\mathbf{E}_{\mathbf{0}}\right|^{4}} .
$$

As shown in Figure 6 in main text, the nm-tAUPs and $\mu$ m-tAUPs show smaller enhancement compared to the rough nm-tAUP structures. According to simulation data, rough tAUPs exhibit much higher electric field enhancement and more spots for enhancement due to increasing numbers of nanospikes. This explains why rough tAUPs have the highest SERS enhancement.

It is worth mentioning that the enhancement here is a local enhancement factor and indicates the enhancement effect at each single location. The total enhancement will be an area integration of the local enhancement factor multiplied by the molecular distribution as discussed in the main text. 


\section{Explanation of the estimation of the number of 4-MBA molecules excited for enhanced Raman scattering.}

Laser spot size: Theoretical laser spot diameter can be estimated using the simplified equation:

$$
S=\frac{1.22 \lambda}{N . A}
$$

where $\mathrm{S}=$ diameter of the laser spot size (beam diameter).

$\lambda=$ wavelength of the laser beam (785 $\mathrm{nm}$ used in this study).

N.A $=$ Numerical aperture $(0.4$ for $20 \times$ objective used in this study (Leica)

Hence, the diameter of spot size $S=1.22 .0 .785 / 0.4=2.39 \mu \mathrm{m}$. The radius of the spot size $=1.19$ $\mu \mathrm{m}$; therefore the illuminated spot area $=1.19 \times 1.19 \times 3.14=4.47 \mu \mathrm{m}^{2}$

Number of 4-MBA molecules excited by Raman scattering:

After casting $5 \mu \mathrm{l}$ of $1 \mathrm{mM}$ ethanolic 4-MBA solution onto tAUP-covered substrates, the observed 4-MBA sample spot radius is $\sim 1 \mathrm{~cm}$, giving a surface area of the sample spot of $3.14 \mathrm{~cm}^{2}$. Then, the total number of 4-MBA molecules on the substrate is $1 \mathrm{mM} \times 5 \mu \mathrm{l}=5 \times 10^{-9}$ moles. The number of molecules interacting with the hotspots on the tAUP nanospikes can be estimated by the area of the nanospikes relative to that of the substrate (Figure S3) $\left(\sim 471 \mathrm{~nm}^{2}\right.$ for roughly 10 spikes). Therefore, the estimated number of 4-MBA molecules excited in the Raman experiment is $7.5 \times 10^{-}$ ${ }^{21}$ moles, which is $\sim 4.52 \times 10^{3}$ molecules. 


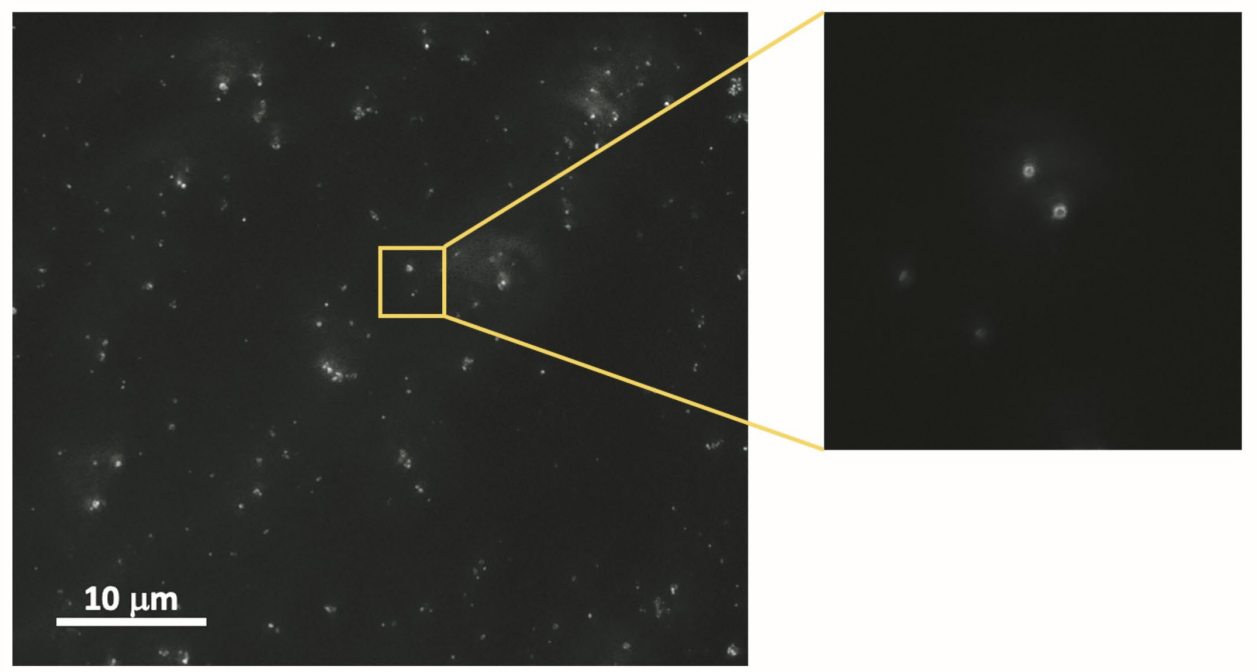

Figure S8. Microscope images of the SERS substrate prepared from tAUPs (micron-sized tAUPs in this case). The inset picture shows individual tAUPs located on the silicon substrate. 


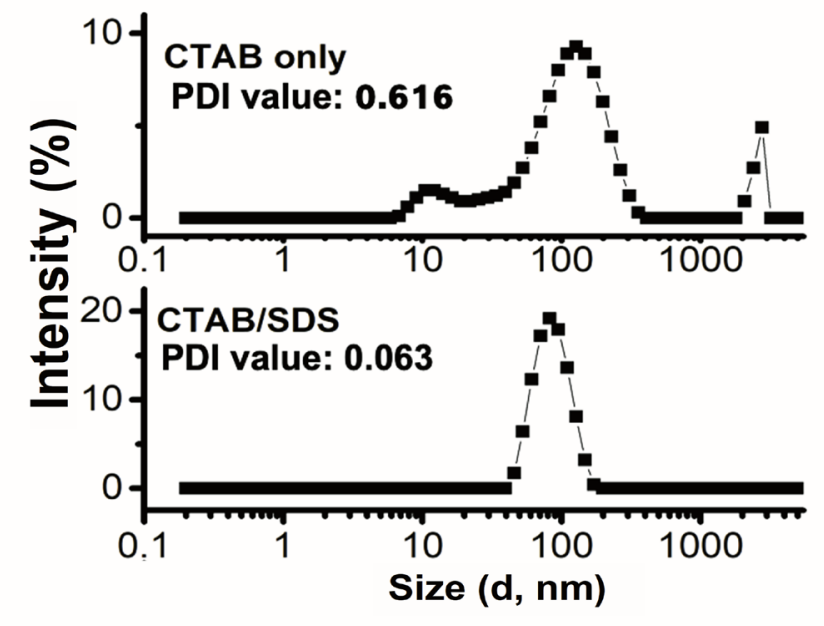

Figure S9. Dynamic Light Scattering (DLS) measurement of CTAB in (a) the absence and (b) the presence of SDS. 


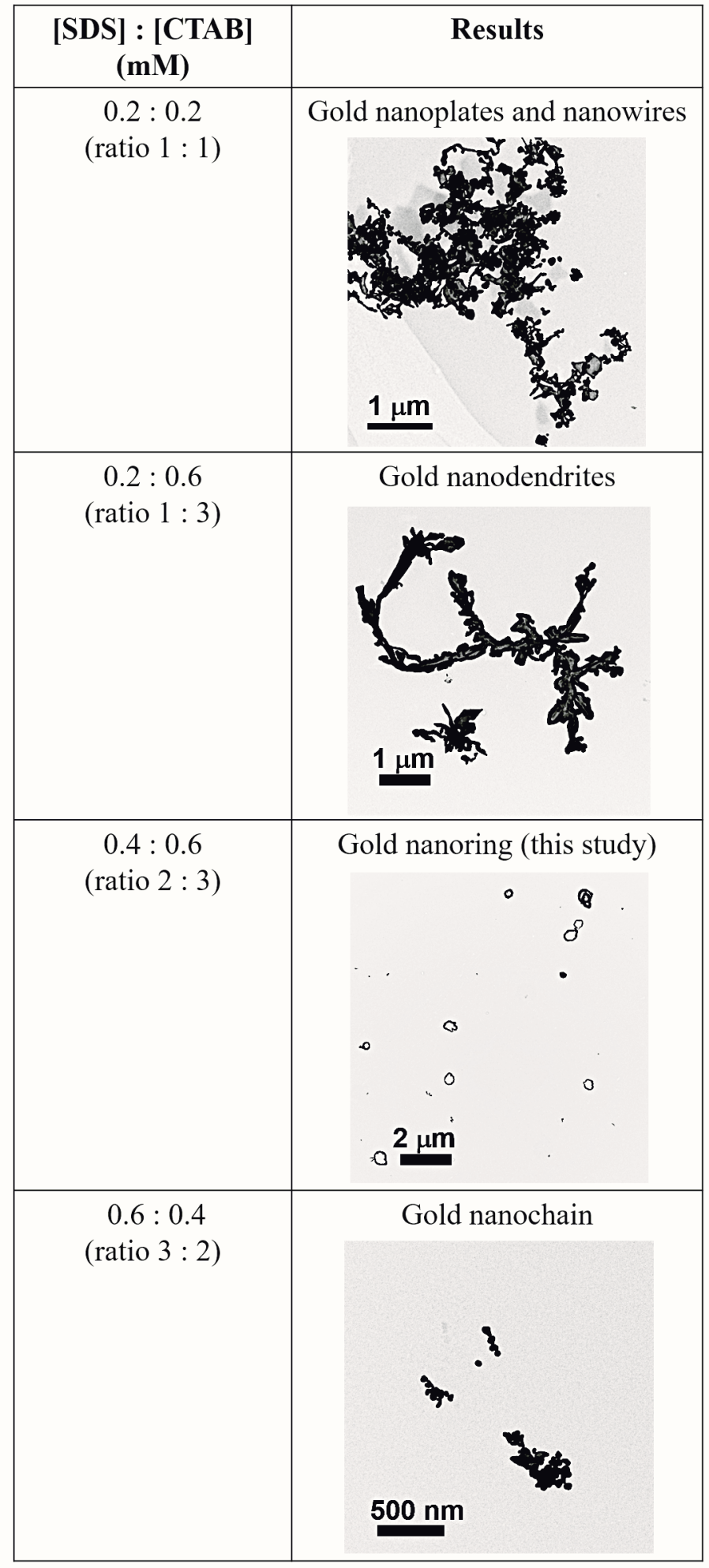

Figure S10. TEM images of gold structures obtained with varying SDS:CTAB ratios. 


\section{$5 \mu \mathrm{l}$ of $5 \mathrm{mM}$ MBA solution prepared in methanol}

(a)

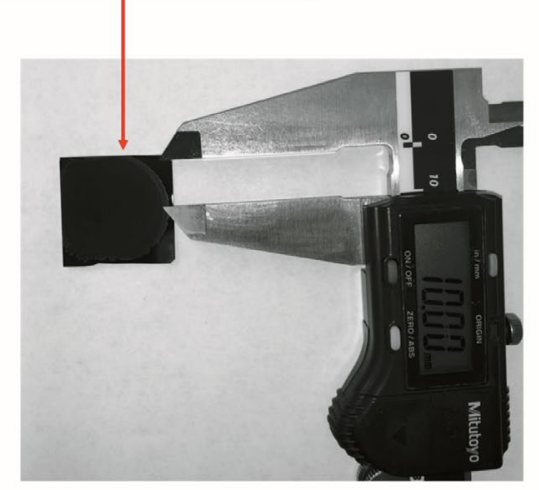

(b)

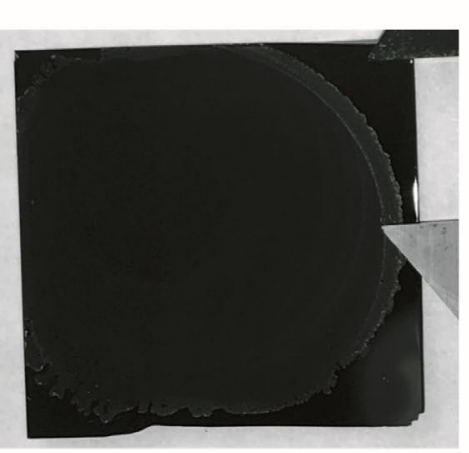

Figure S11. Image of 4-MBA spot size. A drop of 4-MBA (5 mM, prepared in methanol) was placed on a cleaned silicon wafer. The estimated 4-MBA spot size radius was measured using calipers and is approximately $1 \mathrm{~cm}$. 


\section{REFERENCES}

s1. Tsoulos, T.V., Han, L., Weir, J., Xin, H.L., Fabris, L. Nanoscale, 2017, 9, 3766-3773.

s2. Tran, V. Thiel, C. Svejda, J.T., Jalali, M., Walkenfort, B., Erni, D., Schlucker, S. Nanoscale, 2018, 10, 21721-21731.

s3. Kerker, M.; Wang, D.-S.; Chew, H. Surface enhanced Raman scattering (SERS) by molecules adsorbed at spherical particles, Applied Optics, 1980, 19, 4159. https://doi.org/10.1364/AO.19.004159

s4. Kneipp, K.; Kneipp, H.; Itzkan, I.; Dasari, R.R.; Feld, M.S. Surface-enhanced Raman scattering and biophysics, J. Phys.: Condens. Matter, 2002, 14, R597-R624.

s5. Moskovits M. Surface-enhanced spectroscopy. Reviews of modern physics, 1985, 57, 783.

s6. Maier, S.A.; Plasmonics: Fundamentals and Applications (Springer, 2007). 